\title{
The influence of surface tension upon trapped waves and hydraulic falls
}

\author{
C.Page $^{a}$, S.Grandison ${ }^{\mathrm{a}}$, E.I.Părău ${ }^{\mathrm{b}, *}$ \\ ${ }^{a}$ School of Computing Sciences, University of East Anglia, Norwich NR4 7TJ, UK \\ ${ }^{b}$ School of Mathematics, University of East Anglia, Norwich NR4 7TJ, UK
}

\begin{abstract}
We consider steady two-dimensional free-surface flows past submerged obstructions on the bottom of a channel. The flow is assumed to be irrotational, and the fluid inviscid and incompressible. Both the effects of gravity and surface tension are considered. Critical flow solutions with subcritical flow upstream and supercritical flow downstream are sought using fully nonlinear boundary integral equation techniques based on Cauchy integral formula. When a second submerged obstruction is included further upstream in the flow configuration in the absence of surface tension, solutions which have a train of waves trapped between the two obstacles before the critical flow have already been found (Dias and Vanden-Broeck 2004). We extend this work by including the effects of surface tension. Trapped wave solutions are found upstream for small values of the Bond number, for some values of the Froude number. Other types of trapped waves are found for stronger tension when the second obstruction is placed downstream of the hydraulic fall generated by the first obstacle.
\end{abstract}

Keywords: gravity-capillary waves, hydraulic falls, free-surfaces, water waves

\section{Introduction}

The problem of a free-surface flow past a disturbance in a channel, is a widely studied area in fluid mechanics. There are many naturally occurring physical situations that such a problem can model, as the disturbance can be one of many forms: a fully submerged obstruction at the bottom of the channel, e.g. the flow generated by a rock on a river bed, a submerged obstruction not touching the channel bottom such as a submarine moving under water, a surface piercing obstruction, e.g. the flow due to a ship moving through water, or a localised pressure distribution on the free-surface (see Părău and Vanden-Broeck [1]), e.g. atmospheric disturbances caused by high winds. Both steady and unsteady

\footnotetext{
* Corresponding author

Email address: e.parau@uea.ac.uk (E.I.Părău)
} 
solutions to this problem are known to exist, but we consider just the steady case. We concentrate our attention on flow past submerged obstructions.

Solutions for the pure gravity wave problem have been obtained using fully nonlinear methods. In the case of a single submerged obstacle on the bottom of the channel, four different types of basic solutions are known to exist (Dias and Vanden-Broeck [2]). These solutions depend on the Froude number F, defined by

$$
F=\frac{U}{\sqrt{g H}}
$$

where $U$ is the downstream velocity of the fluid, $g$ is the gravitational acceleration, and $H$ is the downstream depth of the fluid. When $F>1$ the flow is said to be supercritical, and when $F<1$, subcritical. We also define the upstream Froude number $F_{\text {up }}$, by

$$
F_{\text {up }}=\frac{V}{\sqrt{g h}}
$$

where $V$ is the upstream velocity of the fluid, and $h$ is the upstream fluid depth. The first type of basic solution is then classified as having uniform supercritical flow both up and downstream of the obstacle, with a forced solitary wave over the obstruction. If the obstacle itself has vertical symmetry about its centre, the free-surface will then also be symmetric about the obstacle. The second type is subcritical and consists of a uniform flow upstream, with a train of waves downstream of the obstacle. For both these solutions, the mean depth of the fluid in the linearised theory, is the same both up and downstream. Forbes and Schwartz [3] used a boundary integral technique to obtain type one and two solutions for flow over a semi-circular obstruction. Vanden-Broeck [4] then found that flows of type one are not unique. There exist two solutions for particular values of the Froude number; a perturbation from a pure solitary wave, and a perturbation from the uniform stream.

The last two types of flow are critical. The first is a hydraulic fall, which consists of a subcritical uniform flow upstream, with a gradual increase in the Froude number over the obstacle, resulting in supercritical uniform flow downstream. The change in the Froude number from subcritical to supercritical means that that the depth of the fluid decreases over the obstacle as we travel downstream. Forbes [5] computed such a flow configuration over a semi-circular obstacle using boundary integral equation techniques, and Dias and VandenBroeck [6] used a series truncation method to obtain solutions over a triangular obstacle. It was found that as the size of the obstacle increased, the downstream Froude number increased, while the upstream Froude number tended to zero.

Generalised hydraulic falls were first computed numerically by Dias and Vanden-Broeck [2]. These are like hydraulic falls but with a train of waves upstream of the obstacle. However, this final type of solution is unphysical when considered as the free-surface flow over a single obstruction, as it does not satisfy the radiation condition, requiring that there is no energy coming from infinity (and thus, no waves upstream of the obstacle). Hydraulic falls have only been observed with subcritical flow upstream. The direction of the 
flow for the generalised hydraulic fall can therefore not simply be changed, so that the flow upstream is uniform and supercritical, in order to satisfy the radiation condition. However, Dias and Vanden-Broeck [7] have shown that this flow can become physically relevant, when considered as the localised flow over an obstacle, in a configuration involving at least one other obstacle further upstream. The free-surface they obtained is a hybrid solution of the second type of basic flow over the obstacle upstream and the generalised hydraulic fall over the second obstacle. Such solutions over multiple obstructions have also been observed experimentally, for example, by Pratt [8] who found that the length and amplitude of the trapped waves between the two obstacles remains unchanged when the distance between the obstacles is increased. Only the number of waves between the obstacles changes. Pratt also found that the solution is characteristic for different shaped obstacles. The shape of the obstacle affects only the amplitude and wavelength of the trapped waves.

Binder, Dias and Vanden-Broeck [9] used both weakly nonlinear and fully nonlinear techniques to consider the possible configurations for flow past two submerged obstructions. They found solutions which have a train of waves upstream of the first obstacle, but uniform subcritical flow between the obstructions, and supercritical uniform flow downstream of the second obstruction. Although not physically relevant due to violating the radiation condition, they noted that such solutions could be made physically relevant, by introducing further obstacles upstream. Belward [10] looked at the solutions obtained when a second obstacle occurs downstream of the hydraulic fall. A forced solitary wave exists over the downstream obstacle, and Belward found that the speed of the flow is almost entirely determined by the hydraulic fall.

There are fewer studies which include the effects of surface tension. To characterise gravity-capillary waves, we introduce the Bond number

$$
\tau=\frac{T}{\rho g H^{2}} .
$$

Here $T$ is the value of the tension on the free-surface and $\rho$ the density of the fluid. Forbes [11] obtained fully numerical solutions for flow over a semi-circular obstacle when the effects of both gravity and surface tension are included. As well as obtaining solutions of type one, Forbes obtained solutions with a train of capillary waves upstream, and gravity waves downstream. Grandison and Vanden-Broeck [12] also studied this type of solution, and removed the inaccuracies in the solutions caused by truncation of the flow both up and downstream.

Maleewong, Asavanant and Grimshaw [13,14] used both fully and weakly nonlinear methods to examine the forced solitary waves produced by a positively and a negatively orientated single applied pressure distribution. However, they restricted their study to the first type of basic solution, in the presence of a single disturbance. Page, Părău and Grandison [15] then studied gravitycapillary forced solitary waves generated by two localised pressure distributions, and Guayjarernpanishk and Asavanant [16] included the effects of surface tension in their study, and found basic flows of types one, two and three. In this paper, we include the effects of surface tension, and consider types three and 
four of the basic solutions; hydraulic falls and generalised hydraulic falls, as well as the effect of a second disturbance in the channel.

In the next section, we mathematically formulate the problem. The results are presented in Section 3, and in the final section, Section 4, we conclude with a summary of our results.

\section{Formulation}

We consider the free-surface of an inviscid, incompressible fluid flowing along a channel. The flow is assumed to be steady and irrotational, and is subject to gravitational acceleration in the negative $y^{*}$-direction. On the channel bottom there exists one or multiple submerged obstructions. We introduce Cartesian coordinates $x^{*}, y^{*}$ and align the $x^{*}$-axis so that it is parallel to the bottom of the channel, with the $y^{*}$-axis directed vertically upwards, through one of the obstructions.

We let $y^{*}\left(x^{*}\right)=H+\eta^{*}\left(x^{*}\right)$ define the free-surface elevation, and take $y^{*}=$ $B^{*}\left(x^{*}\right)$ to be the function describing the bottom of the channel. The flow is assumed to be uniform in the far field, as $x^{*} \rightarrow \pm \infty$, with constant depth $H$, and constant velocity $U$ downstream, and constant depth $h$ and constant velocity $V$ upstream. The downstream Froude and Bond numbers are then given by equations (1) and (3) respectively. The upstream Froude number is given by equation (2), and we introduce the upstream Bond number

$$
\tau_{\text {up }}=\frac{T}{\rho g h^{2}} .
$$

To continue, we non-dimensionalise by taking $U$ as unit velocity, and $H$ as unit height. Non-starred variables are thus now understood to be dimensionless. We define the dimensionless upstream velocity by $\gamma$, and so using conservation of mass, the dimensionless upstream depth is $\frac{h}{H}=\frac{1}{\gamma}$. The dimensionless fluid system is shown in Figure 1.

The problem is formulated as a system of nonlinear equations in terms of the velocity potential $\phi(x, y)$, which, must satisfy Laplace's equation in the flow domain

$$
\phi_{x x}+\phi_{y y}=0
$$

and the corresponding boundary conditions. The kinematic conditions on the free-surface $y=1+\eta(x)$ and the channel bottom $y=B(x)$ are

$$
\phi_{y}=\phi_{x} \eta_{x} \quad \text { and } \quad \phi_{y}=\phi_{x} B_{x}
$$

respectively. Applying Bernoulli's equation on the free-surface, we obtain the dynamic boundary condition

$$
\phi_{x}^{2}+\phi_{y}^{2}+\frac{2}{F^{2}} y=\frac{2}{F^{2}} \tau \kappa+1+\frac{2}{F^{2}}
$$

where

$$
\kappa=\frac{\eta_{x x}}{\left(1+\eta_{x}^{2}\right)^{\frac{3}{2}}}
$$




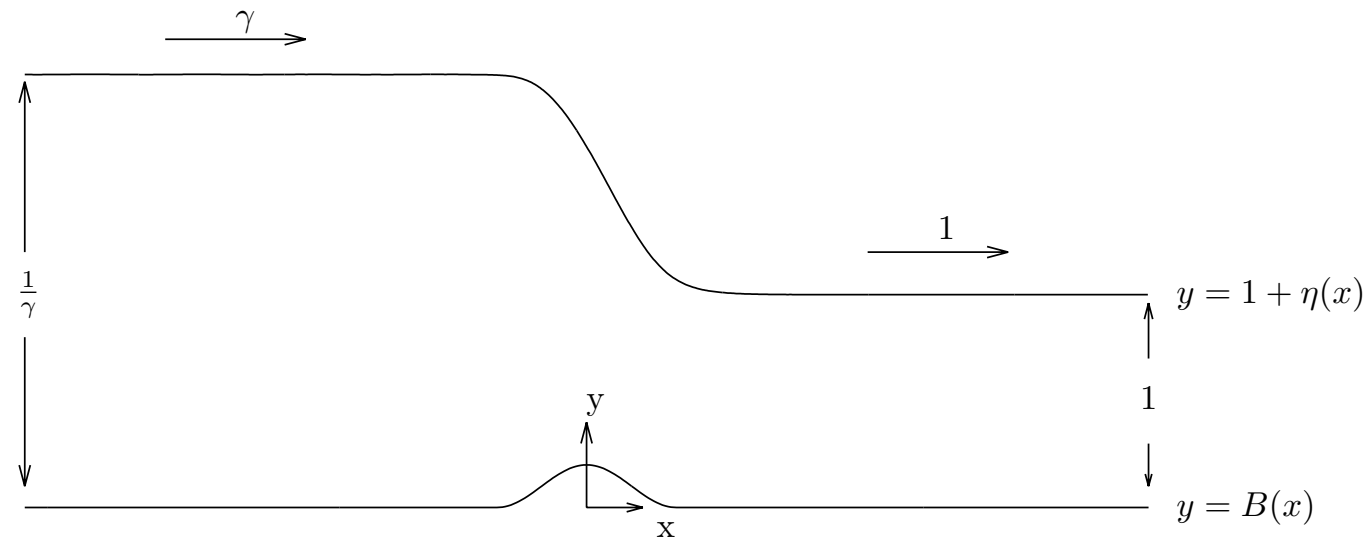

Figure 1: Dimensionless flow configuration over an arbitrary obstacle on the bottom of the channel.

is the curvature of the free-surface at $y=1+\eta(x)$. In the far field, away from the disturbance(s), we require the flow to become that of a uniform stream. Thus, we impose the conditions

$$
\begin{aligned}
& \phi_{x} \rightarrow 1, \quad y(x) \rightarrow 1 \quad \text { as } x \rightarrow \infty, \\
& \phi_{x} \rightarrow \gamma, \quad y(x) \rightarrow \frac{1}{\gamma} \text { as } x \rightarrow-\infty .
\end{aligned}
$$

From conservation of mass, and by applying Bernoulli's equation in the far field both up and downstream (as $x \rightarrow \pm \infty$ ), we obtain the equation

$$
\frac{1}{2}-\frac{1}{2} \gamma^{2}+\frac{1}{F^{2}}-\frac{1}{F^{2} \gamma}=0
$$

Following Forbes [5], a relationship between the upstream and downstream Froude numbers in terms of the non-dimensionalised upstream velocity, can then be found

$$
F_{\text {up }}=F \gamma^{\frac{3}{2}}=\sqrt{\frac{2 \gamma^{2}}{(\gamma+1)}}
$$

and similarly a relationship between the upstream and downstream Bond numbers is given by

$$
\tau_{\text {up }}=\tau \gamma^{2}
$$

Two different types of solution therefore exist. From (11) these solutions are found to correspond to solutions with a constant mean depth both up and downstream, $\gamma=1$, and solutions with a drop in the mean depth of the flow; hydraulic falls, corresponding to

$$
\gamma=\frac{-1+\sqrt{1+\frac{8}{F^{2}}}}{2} .
$$


Following Belward and Forbes [17, 18], Belward [10], and Dias and VandenBroeck [7], we reformulate the problem as a system of integro-differential equations, which can be solved for the unknown free-surface $y=1+\eta(x)$, over arbitrarily shaped obstructions on the bottom of the channel. We parametrise the free-surface by writing $x=X(s), y=Y(s)$, where $s$ is the arclength. We must then satisfy the parametric equation

$$
X^{\prime 2}+Y^{\prime 2}=1
$$

on the free-surface, where the prime denotes differentiation with respect to s. The dynamic boundary condition (7) can be rewritten as

$$
\left(\frac{d \phi}{d s}\right)^{2}+\frac{2}{F^{2}}(Y(s)-1)=\frac{2}{F^{2}} \tau \kappa+1
$$

where $\kappa=Y^{\prime \prime} X^{\prime}-X^{\prime \prime} Y^{\prime}$ is the parametrised curvature of the free-surface.

We introduce the complex variable $z=x+i y$, and the complex potential $w(z)=\phi(x, y)+i \psi(x, y)$, where $\psi(x, y)$ is the stream function. After parametrising, we apply Cauchy's integral formula to the function

$$
\frac{d w}{d z}-\gamma=\phi_{x}-\gamma-i \phi_{y}
$$

around a contour $\mathcal{C}$ consisting of the free-surface, the channel bottom and two vertical lines $x= \pm \infty$. Here $\frac{d w}{d z}$ is the conjugate complex velocity. With the evaluation point $s$ on the free-surface, and using the kinematic conditions (6), we take the imaginary part of the Cauchy integral formula, and obtain the integro-differential equation

$$
\begin{aligned}
& \pi\left(\phi^{\prime}(s) X^{\prime}(s)-\gamma\right) \\
= & -\int_{-\infty}^{\infty} \frac{\left(\phi^{\prime}(\sigma)-\gamma X^{\prime}(\sigma)\right)(Y(s)-Y(\sigma))-\gamma Y^{\prime}(\sigma)(X(\sigma)-X(s))}{(X(\sigma)-X(s))^{2}+(Y(\sigma)-Y(s))^{2}} d \sigma \\
& +\int_{-\infty}^{\infty} \frac{\left(u(\sigma)\left(1+B_{x}(\sigma)^{2}\right)-\gamma\right)(Y(s)-B(\sigma))-\gamma B_{x}(\sigma)(\sigma-X(s))}{(\sigma-X(s))^{2}+(B(\sigma)-Y(s))^{2}} d \sigma
\end{aligned}
$$

and similarly, when the evaluation point, $x$, lies on the channel bottom

$$
\begin{aligned}
& \pi(u(x)-\gamma)=-\int_{-\infty}^{\infty} \frac{\left(\phi^{\prime}(\sigma)-\gamma X^{\prime}(\sigma)\right)(B(x)-Y(\sigma))-\gamma Y^{\prime}(\sigma)(X(\sigma)-x)}{(X(\sigma)-x)^{2}+(Y(\sigma)-B(x))^{2}} d \sigma \\
& +\int_{-\infty}^{\infty} \frac{(-B(\sigma)+B(x))\left(u(\sigma)\left(1+B_{x}(\sigma)^{2}\right)-\gamma\right)-\gamma B_{x}(\sigma)(\sigma-x)}{(\sigma-x)^{2}+(B(\sigma)-B(x))^{2}} d \sigma
\end{aligned}
$$

where $\sigma$ represents the value of the arclength at the varying point $z(\sigma)$ on the contour $\mathcal{C}$, and $u(x)$ is the velocity of the fluid on the channel bottom. Equations (18) and (19) have Cauchy principal value singularities at $\sigma=s$ and $\sigma=x$ in the first and second integrals respectively. 
The two integral equations (18) and (19), together with the dynamic condition (16) and the parametric equation (15), complete the reformulation of the problem, and provide the system of integro-differential equations, to be solved via Newton's method.

Following the work of Dias and Vanden-Broeck [7, 19], the system of integrodifferential equations is solved numerically. To obtain gravity-capillary critical flows, just two independent dimensionless parameters are needed to uniquely determine a solution. We take these to be the obstacle size, and the downstream Bond number $\tau$. The downstream Froude number $F$, and the depth of the channel upstream, are therefore found as part of the solution.

\section{Results}

The results in this section were computed using the numerical method described in Dias and Vanden-Broeck [7]. Following Dias and Vanden-Broeck, a cosine squared profile for the submerged obstructions, of the form

$$
B(x)= \begin{cases}2 A_{1} \cos ^{2}\left(\frac{\pi\left(x+x_{d}\right)}{2 L_{1}}\right) & -L_{1}<x+x_{d}<L_{1} \\ 2 A_{2} \cos ^{2}\left(\frac{\pi x}{2 L_{2}}\right) & -L_{2}<x<L_{2} \\ 0 & \text { otherwise }\end{cases}
$$

is used. The heights of the obstacles are thus given by $2 A_{1}$ and $2 A_{2}$, and the half-lengths of the obstacles by $L_{1}$ and $L_{2}$ respectively. The obstacle of height $A_{2}$ has been chosen such that it is centred at the origin and is of distance $x_{d}$ from the other obstacle. The separation constant $x_{d}$ is negative if the second obstacle occurs downstream where the flow is supercritical, and positive if it is positioned upstream with subcritical flow. The obstacle height $A_{1}$ is taken to be zero when we consider flows with just a single submerged obstruction.

To ensure that our results are numerically accurate we computed the same solutions on meshes of different densities and sizes. By varying the number of mesh points on both the free-surface between $N=401,601,801,1601$, and the channel bottom, between $M=201,301,401$ for a given fixed domain, as well as the mesh spacing $e$ on the free-surface and $h$ on the channel bottom between $e=0.025,0.5,0.1$ and $h=0.05,0.1,0.2$, we were able to determine that our solutions are independent of the mesh for small enough $e$ and $h$. Fewer points were needed to describe the channel bottom accurately than on the free-surface. Further, results were computed for different length domains of the same mesh density where appropriate, to ensure that any errors caused by truncating the integrals, did not impact on the solution.

\subsection{Hydraulic Falls}

Firstly, we consider the case where $A_{1}=0, A_{2}>0$, and fix the value of $L_{2}$, so that $y=B(x)$ describes a uniform channel bottom with a single obstacle. For a pure gravity flow, $\tau=0$, we obtain the hydraulic fall solutions previously computed by Dias and Vanden-Broeck [7], where the flow upstream is subcritical 


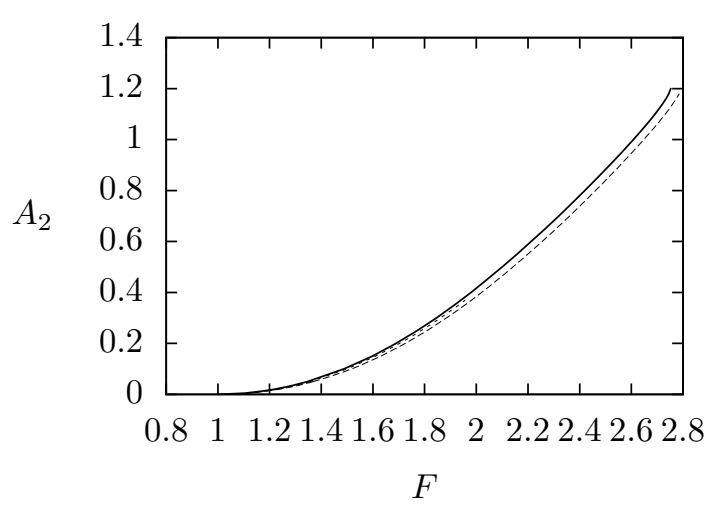

(a)

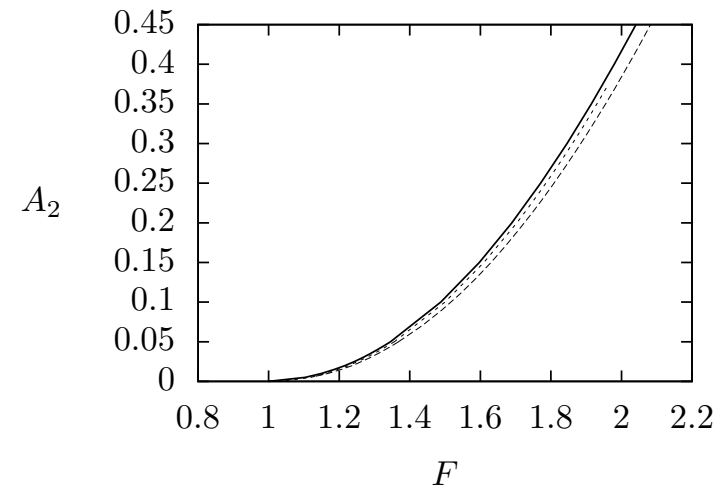

(b)

Figure 2: Relationship between the downstream Froude number $F$, and the half-height $A_{2}$ of the submerged obstacle of half-width $L_{2}=3.2$. The solid curve represents the relationship for $\tau=0$, the dotted curve is the relationship for $\tau=0.2$, and the dashed broken curve is the relationship for $\tau=0.5$. (b) is a close-up of (a), showing the $\tau=0.2$ curve.

and uniform, and the flow downstream is supercritical and uniform. A typical hydraulic fall profile without tension can be seen in Figure 3 of Dias and VandenBroeck [7]. As shown by Forbes [5], as the height of the obstacle is increased, the speed of the flow downstream increases, and thus the height of the channel upstream increases. The relationship between the Froude number and the height of the forcing obstacle, for $L_{2}=3.2$ fixed, can be seen by the solid line in Figure 2. Very small amplitude waves begin to appear on the free-surface upstream as the obstacle height is increased. Such waves have previously been explained by Forbes and Schwartz [3] as being a result of the numerical scheme requiring that the flow is uniform at the first mesh point upstream, rather than at negative infinity. We obtained solutions over very tall obstructions, thus, allowing for very fast flows downstream.

When the effects of surface tension are included, we find similar hydraulic fall profiles with subcritical uniform flow upstream and supercritical uniform flow downstream. Figure 3 shows typical solution profiles for the fixed parameters $A_{2}=0.05, L_{2}=3.2$ and three different values of the Bond number; weak tension both up and downstream $\tau=0.32$, strong tension downstream $\tau=0.6$ with weak tension upstream, and strong tension both up and downstream $\tau=0.9$.

For $0<\tau<\tau^{*}$, where $\tau^{*}$ is some critical value of the Bond number, increasing the strength of the tension, increases the Froude number $F$, and thus increases the height of the channel upstream. The gradient of the hydraulic fall is also found to increase with the tension. The relationship between the downstream Froude number and the downstream Bond number for $0.175<\tau<5$ with $A_{2}=0.05, L_{2}=3.2$ can be seen in Figure 4 . At the critical Bond number $\tau^{*} \approx 1.403$ with corresponding $F^{*} \approx 1.397$ there exists a turning point in the 


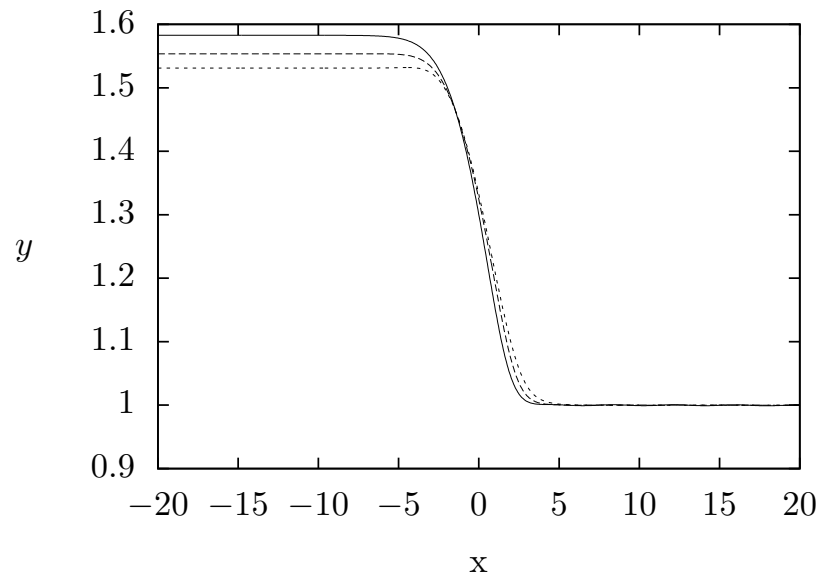

Figure 3: Hydraulic fall solutions, including the effects of surface tension, over a single submerged obstruction of height $2 A_{2}=0.1$ and half-width $L_{2}=3.2$. The solid line is the solution with $\tau=0.9, F=1.393$, the dashed line the solution with $\tau=0.6, F=1.375$ and the dotted line the solution with $\tau=0.32, F=1.361$. In each case the Froude number was found as part of the solution.

$F-\tau$ plane, and thus, it is possible to obtain more than one solution with the same value of the Froude number $F$ and the same obstruction $\left(A_{2}, L_{2}\right.$ fixed), but with different values of the Bond numbers. Figure 5 shows an example of this, with two hydraulic falls over the same obstacle, with the same Froude number $F=1.398$, but different values of the tension $\tau=1.024$ and $\tau=2$. Further, there exists a hydraulic fall with tension, which has the same Froude number as the pure gravity case, and the same upstream depth, but with a steeper fall.

We can explain the possibility of multiple solutions for a fixed value of the Froude number, by considering the linear theory. Substituting a linear periodic wave function into the linearised versions of the governing equations (5), (6) and (7), we obtain the dispersion relations

$$
\begin{array}{r}
F_{\text {up }}^{2}=\left(\frac{\gamma}{k}+\frac{\tau_{\text {up }}}{\gamma} k\right) \tanh \left(\frac{k}{\gamma}\right) \\
F^{2}=\left(\frac{1}{k}+\tau k\right) \tanh (k)
\end{array}
$$

up and downstream respectively. Here $k=K H$, where $K$ is the wavenumber of the waves. For a fixed Froude number $F$, there is just a single dispersion curve possible for the pure gravity case, given by equations (21) and (22) with $\tau=\tau_{\text {up }}=0$. However, when we include surface tension, we are free to change the value of the Bond number $\tau$ for any given $F$, and thus multiple different dispersion curves can exist for the same fixed $F$, allowing for multiple solutions.

For small $\tau$, small numerical oscillations appear on the solution branch in the 


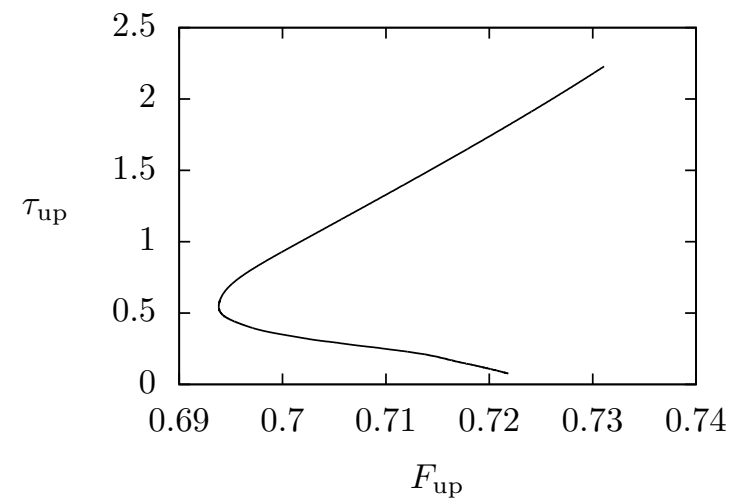

(a) Upstream

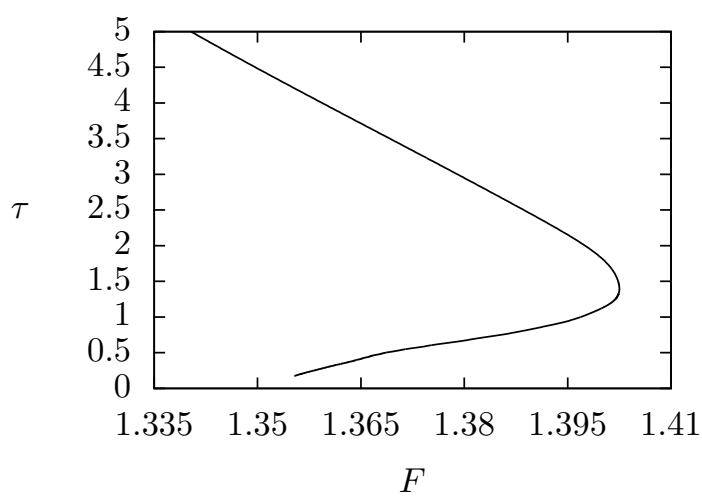

(b) Downstream

Figure 4: Relationship between the Bond and Froude number both up and downstream for a single submerged obstacle characterised by $A_{2}=0.05, L_{2}=3.2$.

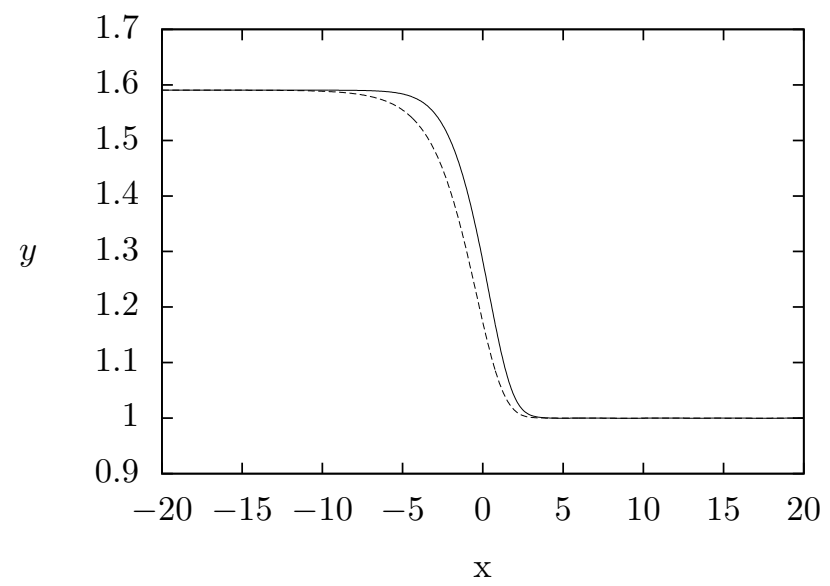

Figure 5: Hydraulic fall solutions, including the effects of surface tension, over a single submerged obstruction of height $2 A_{2}=0.1$ and half-width $L_{2}=3.2$, with Froude number $F=1.398$. The solid line is the solution with $\tau=1.024$, and the broken line is the solution with $\tau=2$. In both cases the Froude number was found as part of the solution. 


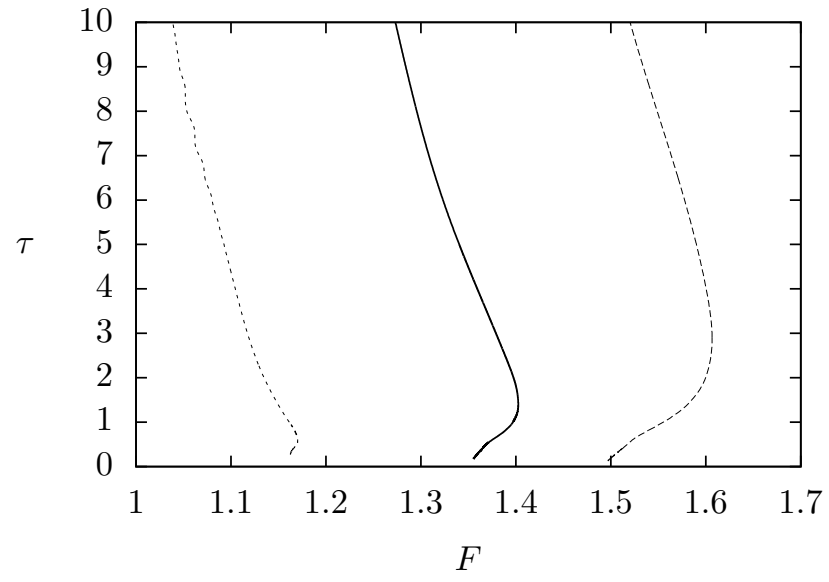

Figure 6: Relationship between the Bond and Froude number downstream for a single submerged obstacles with $L_{2}=3.2$. The dotted line is the branch with $A_{2}=0.01$, the solid line the branch with $A_{2}=0.05$ and the dashed line the branch with $A_{2}=0.1$.

$F-\tau$ plane. More mesh points are needed for the required degree of accuracy, but the computation cost then becomes prohibitive. Also for small $\tau$, as $\tau$ is decreased, the upstream Froude number $F_{\text {up }}$ approaches the minimum of the upstream dispersion curve. We end the branch in the $F-\tau$ plane at $\tau=0.175$ where $F_{\text {up }}$ is just below the minimum, as above the minimum, the linear theory then suggests the presence of some capillary waves upstream, which would be eliminated by the imposition of a flat upstream in our scheme.

For different sized obstacles, similar solution branches can be obtained in the $F-\tau$ plane. Figure 6 shows the solution branches for $A_{2}=0.01, A_{2}=0.05$ and $A_{2}=0.1$ with $L_{2}=3.2$ fixed, for $\tau<10$. The value of the critical Bond number increases with $A_{2}$, so that, for an obstacle of height $A_{2}=0.1$, for example, $\tau^{*} \approx 1.606$ and the corresponding value of the downstream Froude number is $F^{*} \approx 2.894$. As the size of $\tau$ increases, numerical oscillations begin to appear on the solution branch, as can be seen on the $A_{2}=0.01$ branch in Figure 6. Again, more points on both the free-surface and the channel bottom are therefore needed to obtain the required degree of accuracy.

As the height of the obstacle increases, the downstream Froude number increases whilst the upstream Froude number decreases, for a given uniform flow. This is comparable to the findings of Forbes [5] for the pure gravity case. The relationship between $F$ and $A_{2}$ can be seen in Figure 2 for $\tau=0.2$ and $\tau=0.5$. Guayjarernpanishk and Asavanant [16] found that critical flow solutions do not exist when surface tension is present, for obstacles below some critical height. Further, they found that as the height of the obstacle decreases towards the critical height, with $\tau_{\text {up }}$ fixed, the free-surface immediately before the hydraulic fall develops a slight elevation. Similarly, for an obstacle of fixed height, as the Bond number is decreased towards some critical value, the slight 


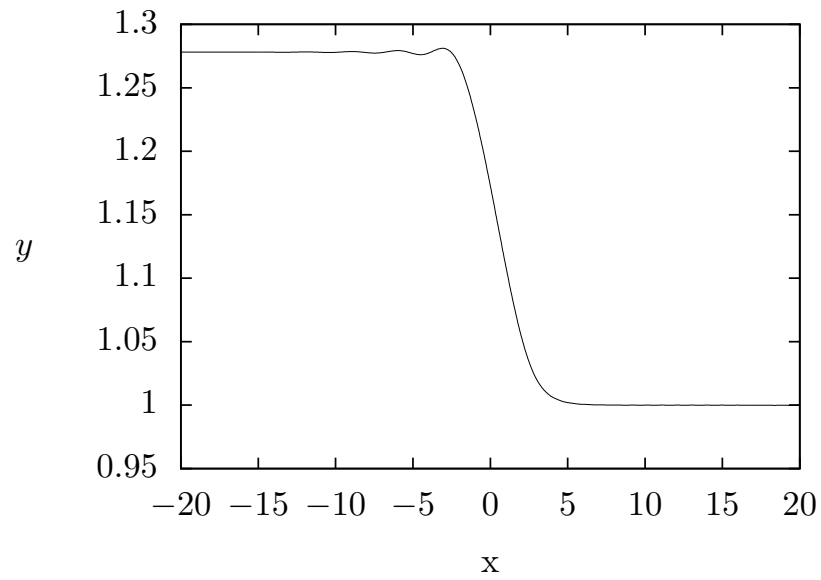

Figure 7: Hydraulic fall solution over a single submerged obstruction characterised by $A_{2}=$ $0.015, L_{2}=3.2$. Weak surface tension acts on the free-surface; $\tau=0.2$. A small train of decaying waves immediately before the hydraulic fall can be seen. The Froude number $F=1.198$ is found as part of the solution.

elevation immediately before the hydraulic fall, increases.

When $\tau_{\text {up }}$ is weak $\left(\tau_{\text {up }}<\frac{1}{3}\right)$, the upstream dispersion curve possesses a minimum $F_{\text {up }_{\min }}<1$. As we decrease $A_{2}$, the upstream Froude number $F_{\text {up }}$ increases towards $F=1$, and thus approaches the minimum of the dispersion curve. We obtain similar results to Guayjarernpanishk and Asavanant and find that for $F_{\text {up }}$ near $F_{\text {up }_{\min }}$, the free-surface immediately before the hydraulic fall develops a slight elevation. As we continue to decrease $A_{2}$, the amplitude of the slight elevation increases, and we have found solutions where the elevation becomes part of a small train of decaying waves immediately before the hydraulic fall. An example free-surface profile is shown in Figure 7 with $\tau=0.2$ and $F=$ 1.198. At some critical value of $A_{2}$, the Froude number intersects the dispersion curve, and we cease to obtain accurate solutions. Numerically generated waves start to appear on the free-surface.

For stronger tension, $\tau_{\mathrm{up}}>\frac{1}{3}$, the upstream dispersion curve increases monotonically. As we decrease $A_{2}, F_{\text {up }}$ increases towards $F=1$, and we continue to obtain hydraulic fall solutions until the solution becomes that of a uniform stream.

The maximum height of the obstacle for which we can find solutions, is also determined by the surface tension $\tau$. The maximum height in the puregravity case, with $\tau=0$, is quite large, but on introducing a small amount of surface tension it reduces rapidly. As the surface tension is then increased, the maximum obstacle height also increases with $\tau$. 


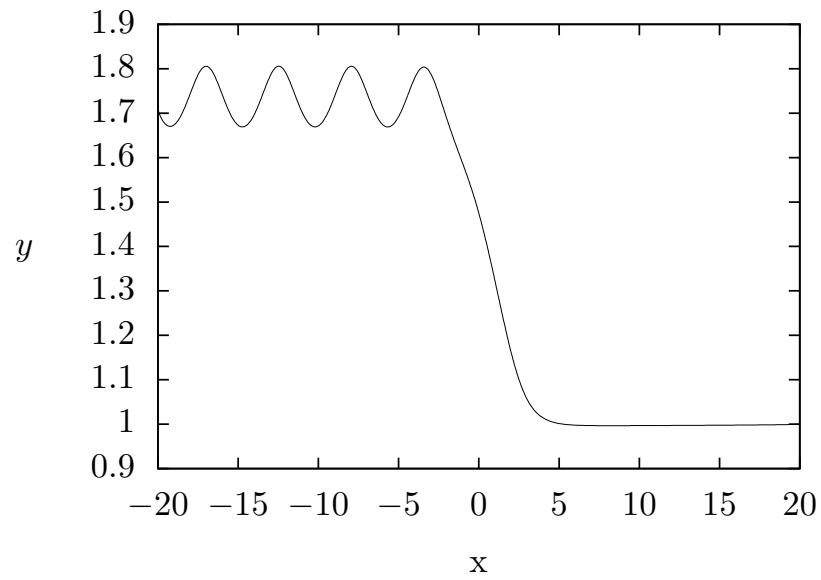

Figure 8: Generalised hydraulic fall solution over a submerged obstruction of height $2 A_{2}=0.2$ and half-width $L_{2}=3$. The downstream Froude and Bond numbers are given by $F=1.482$ and $\tau=0.01$ respectively.

\subsection{Generalised Hydraulic Falls}

Following the work of Dias and Vanden-Broeck [7], we modified the numerical scheme, to look for generalised hydraulic falls. Such a flow requires three independent parameters to uniquely define a solution in the pure gravity case. We take these parameters to be the Froude number $F$, the obstacle size, and for convenience, the height of the channel at the first mesh point. Instead of ensuring that the free-surface is flat far upstream as $x \rightarrow-\infty$, we impose the condition

$$
Y(1)=\delta
$$

for some given constant $\delta$, and fix the value of $F$. By varying $\delta$ we were able to compute different generalised hydraulic falls, in the absence of surface tension. Typical generalised hydraulic fall profiles with $A_{2}=0.4$ and $L_{2}=3.2$ can be seen in Figure 4 of Dias and Vanden-Broeck [7], for two different values of $\delta$. However, following this scheme, we were only able to obtain gravity-capillary generalised hydraulic falls if the surface tension was very weak.

We explain this result by considering the linear dispersion relations both up and downstream, given by equations (21) and (22). When the tension is such that $\tau_{\text {up }}<\frac{1}{3}$, the upstream dispersion curve possesses a minimum. We define $F_{\min }$ to be the critical value of the Froude number corresponding to this minimum. The Froude number $F$ may then intersect the dispersion curve, if it is such that $F_{\min }<F<1$. Upstream waves are then possible for a critical range of Froude numbers within this region, resulting in gravity-capillary generalised hydraulic fall solutions. A typical solution is shown in Figure 8 with $\tau=0.01$. For different values of $\delta$ we were able to obtain upstream waves of different amplitudes. 


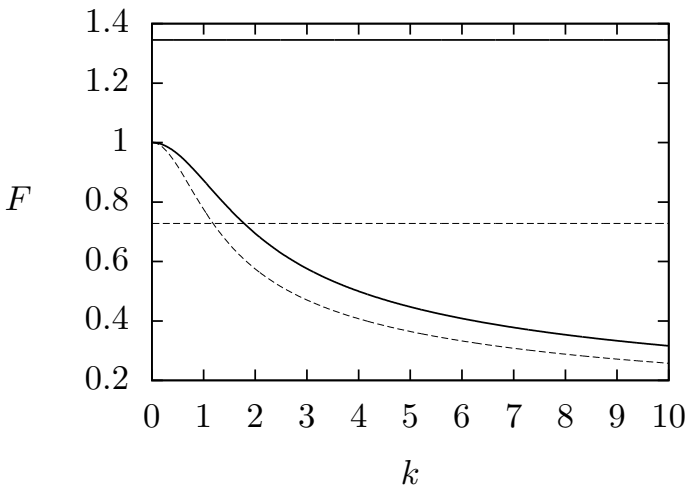

(a) Pure gravity case, $\tau=0$.

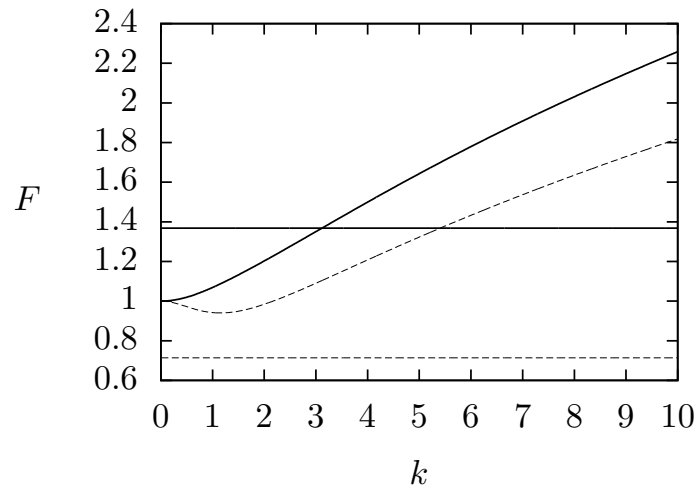

(b) Gravity-capillary case, $\tau=0.5$.

Figure 9: Linear dispersion relations. The solid curves represent the dispersion relation downstream, and the solid constant lines give the downstream value of the Froude number $F$ for a submerged obstacle classified by $A_{2}=0.05, L_{2}=3.2$. The broken curves represent the dispersion relation upstream, and the broken constant lines give the upstream value of the Froude number $F_{\text {up }}$ for the same obstacle.

For stronger surface tension, we were unable to obtain generalised hydraulic falls. Figure $9($ a) shows that the upstream dispersion relation in the pure gravity case (see broken lines) predicts the occurrence of a train of waves upstream of an obstacle of height $2 A_{2}=0.1$ and width $2 L_{2}=6.4$, as the Froude number intersects the dispersion curve. The downstream relation shows that no waves are predicted downstream (see solid lines). When strong surface tension is included however, the situation is reversed. Figure 9(b) shows that linear theory now predicts a train of waves downstream of the fall (see solid lines), with a uniform flow upstream (see broken lines). However, waves appearing downstream of a hydraulic fall would correspond to waves travelling with speeds predicted by the linear dispersion relation, with $\frac{d F}{d k}>0$ where the group velocity is greater than the phase velocity. We would therefore expect such waves to appear in front of, rather than behind, an obstacle. Such solutions would therefore appear to lack physical relevance in a configuration involving just a single obstacle. These solutions may become physically relevant however, when considered as the localised flow over an obstacle in a configuration involving a further obstacle further downstream. This is discussed in the next section.

\subsection{Trapped Waves}

When surface tension is neglected, $\tau=0$, and a second obstruction is included further upstream (so $x_{d}>0$, with $A_{1}>0$ ), a train of waves appears between the two obstacles, with uniform flow upstream of the first obstacle as $x \rightarrow-\infty$, and downstream after the fall, as $x \rightarrow \infty$. Such solutions were found by Dias and Vanden-Broeck [7], and typical profiles can be seen in their Figure 


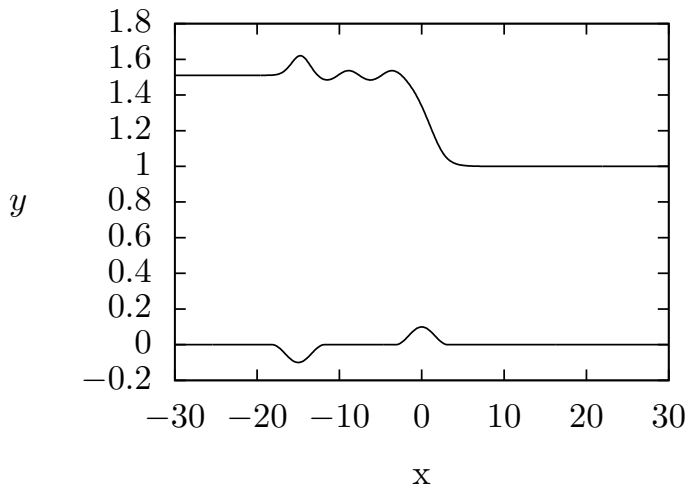

(a)

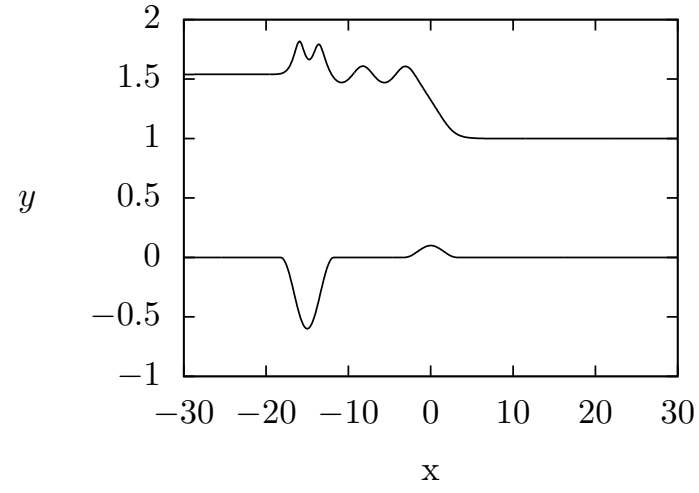

(b)

Figure 10: Free-surface profile past 2 disturbances on the bottom of the channel. The first disturbance represents a dip in the channel, and is given by a) $A_{1}=-0.05, L_{1}=3.2$, b) $A_{1}=$ $-0.3, L_{1}=3.2$, centred upstream at $x=-15$, and the second is an obstacle characterised by $A_{2}=0.05, L_{2}=3.2$. Waves appear trapped between the obstacles. In a) $F=1.348$ and an elevation wave is found over the dip. In b) $F=1.364$ and a two-peaked elevation wave is found over the dip. In both cases, the Froude number was found as part of the solution.

5. They used an argument based on weakly nonlinear theory to show that as the distance between the two obstructions increases, the flow over the obstacle centred at $x=0$, approaches that of a generalised hydraulic fall past a single disturbance. As there are no waves in the far field, the radiation condition is now satisfied. Thus, the unphysical generalised hydraulic fall solution becomes physically relevant when considered as the local flow over an obstacle in a configuration involving a second obstacle further upstream.

For the case with $A_{1}<0$, the hydraulic fall is preceded by a dip in the channel, and a train of trapped waves is again found between the obstacles. Here however, the behaviour of the free-surface over the upstream disturbance changes. An elevation appears over the dip, before the train of waves. For a wide enough dip, this elevation wave can be multi-peaked. Free-surface profiles with single and multi-peaked elevations over the dip in the channel are shown in Figure 10.

Including a second obstacle further upstream when surface tension is present does not result in trapped waves between the obstacles, unless the upstream tension is very small. Dias and Vanden-Broeck [7] have shown that such trapped wave solutions are hybrid solutions between a generalised hydraulic fall, which we have not been able to obtain when the effects of surface tension are considered (unless the tension is very weak), and type two basic solution. It is therefore no surprise that we do not obtain trapped waves upstream for stronger values of $\tau_{\text {up. }}$.

For very weak surface tension, when the upstream dispersion curve possesses a minimum such that the Froude number $F_{\text {up }}$ intersects the dispersion curve (i.e. 


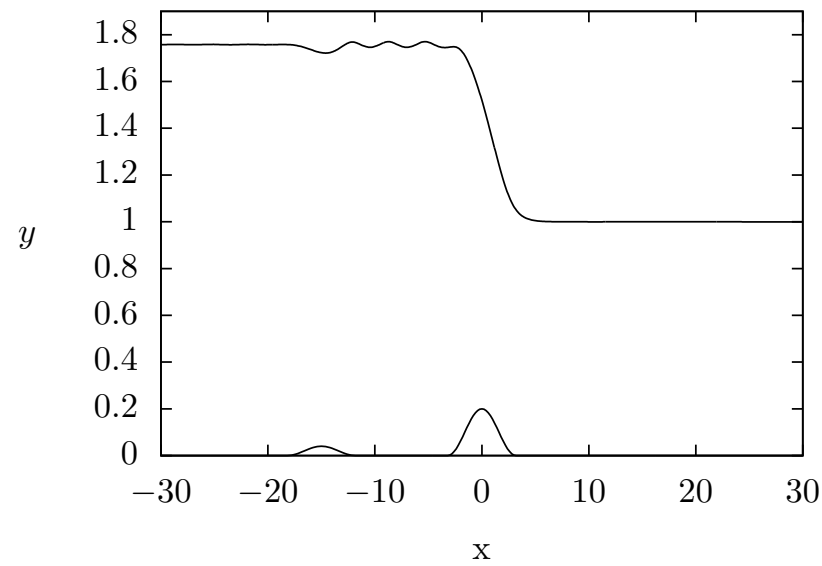

Figure 11: Free-surface profile past 2 obstacles on the bottom of the channel, with weak tension $\tau=0.1$. The first of which is characterised by $A_{1}=0.02, L_{1}=3.2$ and is centred upstream at $x=-15$, and the second by $A_{2}=0.1, L_{2}=3.2$. Small amplitude waves appear trapped between the obstacles. The Froude number $F=1.497$ is found as part of the solution.

$F_{\mathrm{up}_{\min }}<F_{\mathrm{up}}<1, F_{\mathrm{up}_{\min }}$ the value of the Froude number corresponding to the minimum of the dispersion curve), we were able to obtain trapped wave solutions. Figure 11 shows trapped waves between a hydraulic fall and an upstream obstacle, for $\tau=0.1, F=1.497$. The upstream Bond number is thus extremely small, and is given by $\tau_{\text {up }} \approx 0.032$. The linear theory therefore now predicts a train of gravity dominated waves between the obstacles, and a train of capillary dominated waves upstream as $x \rightarrow-\infty$. The capillary wave train is not captured by our numerical scheme, which ensures that the free-surface is flat upstream in order to satisfy the radiation condition. Instead, a train of very small amplitude spurious waves with the same wavelength as the downstream wave train is found. By gradually increasing the tension $\tau_{\mathrm{up}}, F_{\mathrm{up}_{\min }}$ approaches 1 , and so $F_{\text {up }}$ approaches the minimum. At this point, near the minimum, our calculations fail to provide solutions with the required degree of accuracy. As $F_{\text {up }_{\text {min }}}$ increases further, so that $F_{\text {up }}<F_{\text {up }_{\text {min }}}<1$ the Froude number ceases to intersect the dispersion curve, and so the trapped waves disappear. The solutions obtained in this case are discussed in Section 3.4.

Figure 12 shows upstream trapped wave solutions for the pure gravity wave case $\left(\tau_{\mathrm{up}}=0\right)$ and a case including the effects of surface tension $\left(\tau_{\mathrm{up}}=0.04\right)$. We see that the wavelength of the trapped waves in the gravity-capillary solution, is smaller than the wavelength of the pure gravity waves. The linear dispersion relations for both the pure gravity case and the gravity-capillary case with $\tau_{\text {up }}=0.04$ (corresponding to $\tau=0.1$ ) are shown in Figure 13. From the figure we can see that the wavenumber of the gravity-capillary waves is greater than that of the pure gravity waves, and thus this serves to help validate our results.

For stronger tension, we place the second obstacle downstream of the hy- 


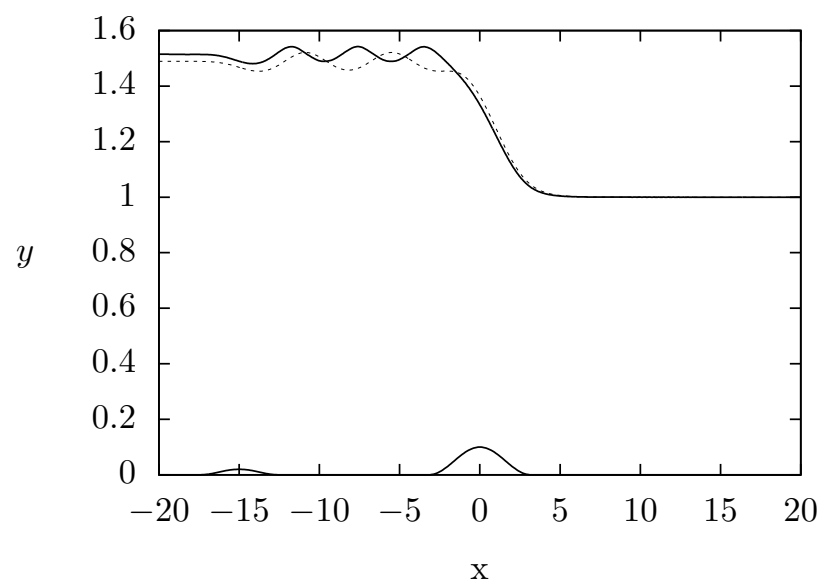

Figure 12: Free-surface profile past 2 obstacles on the bottom of the channel, characterised by $A_{2}=0.05, L_{2}=3.2$ and $A_{1}=0.01, L_{1}=3.2$. The solid curve represents the solution with $\tau=0.1$ and $F=1.350$. The broken curve represents the pure gravity solution with $\tau=0$ and $F=1.334$.

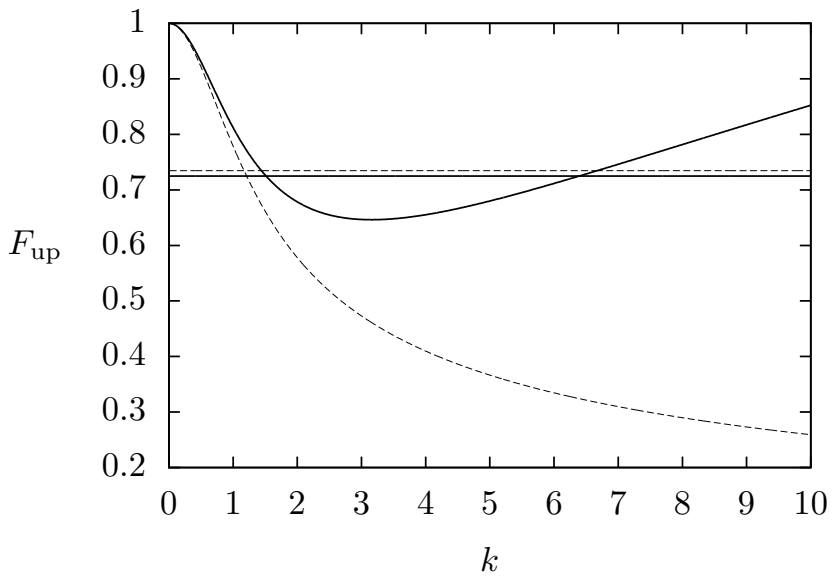

Figure 13: Upstream linear dispersion relations. The solid curves represent the dispersion relation with $\tau=0.1$ and the upstream value of the Froude number $F_{\text {up }}$ for submerged obstacles characterised by $A_{2}=0.05, L_{2}=3.2$ and $A_{1}=0.01, L_{1}=3.2$. The broken curves are the dispersion relation with $\tau=0$ and the upstream value of the Froude number for the same channel bottom configuration. 


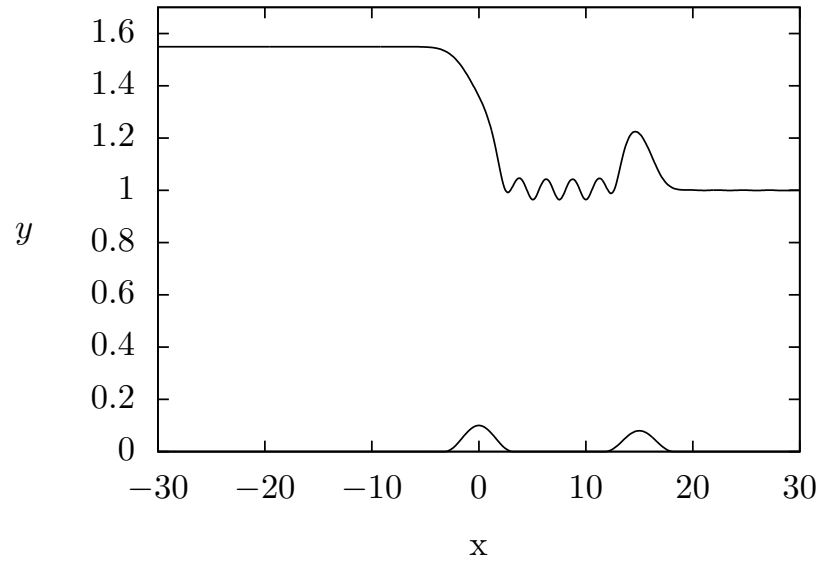

Figure 14: Free-surface profile past 2 obstacles on the bottom of the channel, with surface tension $\tau=0.6$. The first of which is characterised by $A_{2}=0.05, L_{2}=3.2$ and the second by $A_{1}=0.04, L_{1}=3.2$, centred downstream at $x=15$. Trapped waves appear trapped between the obstacles. The Froude number $F=1.372$ is found as part of the solution.

draulic fall, where the flow is supercritical. As might be expected from the linear theory, we find a train of waves trapped between the obstacles. Further downstream, in the far field beyond the obstacles, the flow is uniform and supercritical. Upstream of the first obstacle, the flow is uniform and subcritical. A typical free-surface profile with $\tau=0.6$ is shown in Figure 14. An elevation wave is found over the downstream obstruction.

By increasing $x_{d}$, the distance between the obstacles, the number of waves trapped between the obstacles increases. The amplitude and wavelength of the waves change very little. This is similar to the results of the experiments by Pratt [8] where trapped waves were obtained upstream between 2 obstacles, as in the gravity case. Pratt found that the amplitude and wavelength of the waves remained the same when the position of the obstacles was altered, and just the actual number of trapped waves changed.

We investigated the effect of the downstream obstruction on the waves, by changing its half-height $A_{1}$ whilst keeping $A_{2}, L_{1}, L_{2}$ and $\tau$ fixed. Decreasing the obstacle height decreases the amplitude of the trapped waves. When the downstream obstruction is small compared to the central obstruction, the amplitude of the waves becomes so small, that the waves cease to be visible between the obstructions. The free-surface thus appears to become that of a hydraulic fall with an elevation solitary wave over the downstream obstruction, as can be seen in Figure 15. Such solutions are comparable with the pure-gravity wave solutions obtained by Belward [10] for flow over two successive obstacles. Belward found a hydraulic fall over the first obstacle, and a near symmetric elevation wave in the supercritical flow regime, over the second. However, very small amplitude trapped waves still exist between the obstacles in the gravity-capillary case, with 


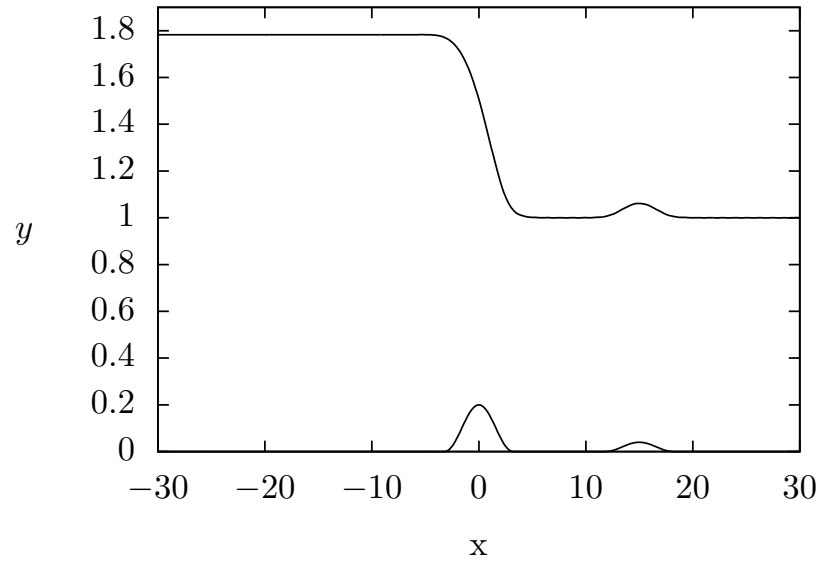

Figure 15: Free-surface profile past 2 obstacles on the bottom of the channel, with surface tension $\tau=0.4$. The first of which is characterised by $A_{2}=0.1, L_{2}=3.2$, and the second $A_{1}=0.02, L_{1}=3.2$, centred downstream at $x=15$. The free-surface appears to be uniform between the obstacles. The Froude number $F=1.512$ is found as part of the solution.

the waves becoming visible upon much closer examination of the free-surface.

In the pure-gravity case, Belward found that the value of the Froude number was almost entirely determined by the height of the first obstacle. By increasing the height of the obstacle, the Froude number increases. For a given value of $\tau$, a similar dependency of the Froude number on the size of $A_{2}$ is found in the gravity-capillary case.

The gravity-capillary trapped wave solution is not unique. For a fixed channel bottom configuration, multiple families of trapped wave solutions with the same value of the Froude number are obtained. Several different solution branches in the $F-\tau$ and the $F_{\text {up }}-\tau_{\text {up }}$ planes for the flow configuration classified by $A_{2}=0.1, A 1=0.05$, with $L_{1}=L_{2}=3.2$ are shown in Figure 16. Six corresponding free-surface profiles with $F=1.53597$ are shown in Figure 17. We see that as $\tau$ increases, with $F$ fixed, the wavelength and amplitude of the trapped waves increases.

The gradient of the hydraulic fall is again determined by the Bond number, with steeper falls occurring for smaller values of $\tau$. As the Bond number increases, the steepness of the associated gravity-capillary linear dispersion curve increases, and thus the wave number of the trapped waves decreases. The linear theory therefore predicts that the wavelength of the trapped waves increases as $\tau$ increases, as we have observed, and thus helps to validate our results.

The effects of surface tension on the waves appear to be more important than those of gravity. The non-linear effects can be seen in the waves, as rather than being a sinusoidal wave train, the waves have slightly more rounded crests than troughs, and thus are dominated by the effects of capillarity. This is visible in Figure 18, which shows high amplitude trapped waves between the obstacles 


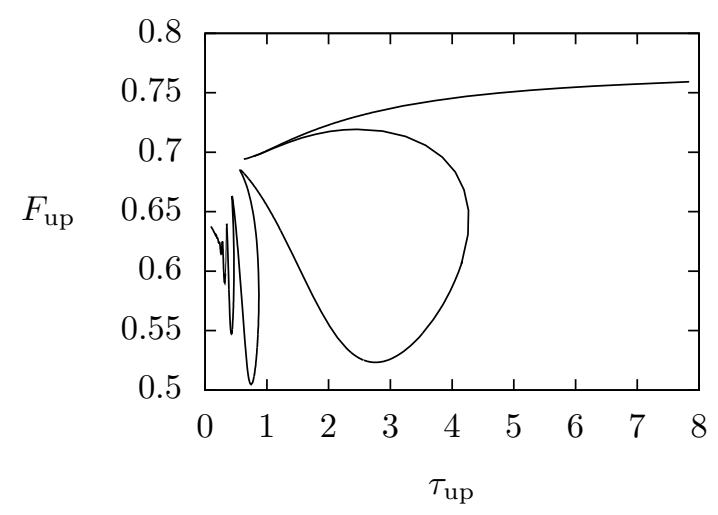

(a) Upstream

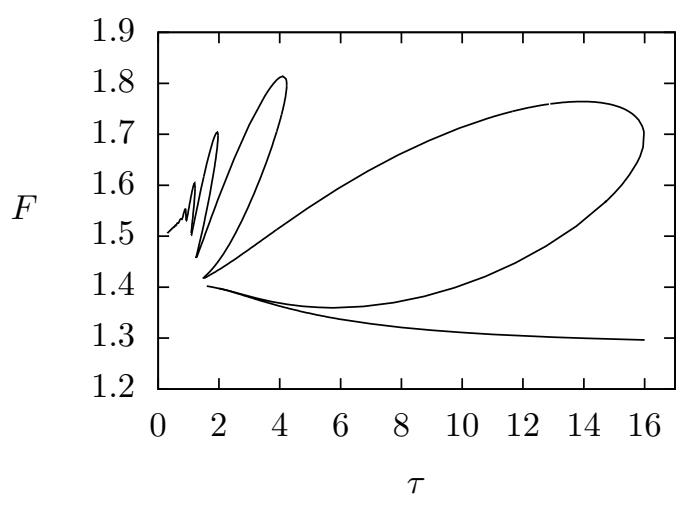

(b) Downstream

Figure 16: Gravity-capillary trapped wave solution branches in a) the $F_{\text {up }}-\tau_{\text {up }}$ plane and b) the $F-\tau$ plane. The two submerged obstacles on the bottom of the channel are classified by $A_{2}=0.1, A_{1}=0.05$ and $L_{1}=L_{2}=3.2$.

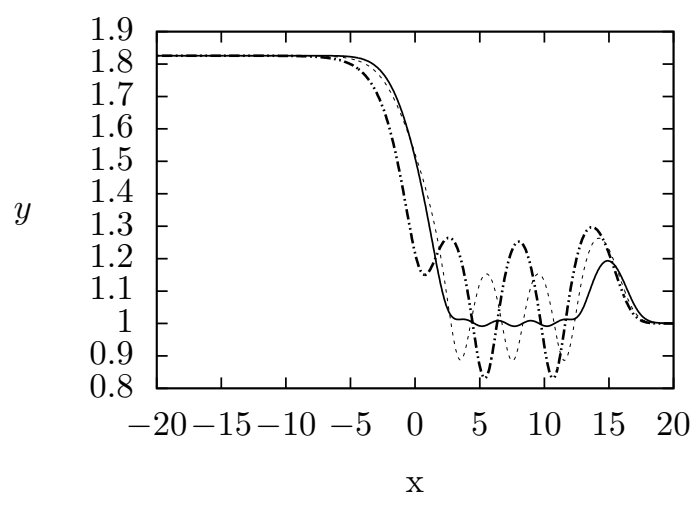

(a) Solutions for $\tau=0.8, \tau=1.2074$ and $\tau=1.54212$

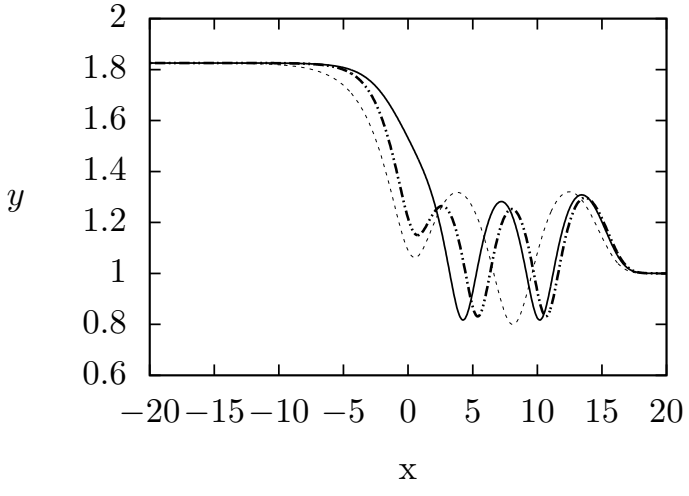

(b) Solutions for $\tau=1.74, \tau=2.973$ and $\tau=4.451$

Figure 17: Free-surface profiles past two obstacles on the bottom of the channel, characterised by $A_{2}=0.1, L_{2}=3.2$, centred at $x=0$ and $A_{1}=0.05, L_{1}=3.2$ centred downstream at $x=15$. Multiple trapped wave solutions are found for the same value of the Froude number $F=1.53597$, but with different values of the Bond number. For clarity, only the central part of the free-surface is shown. 


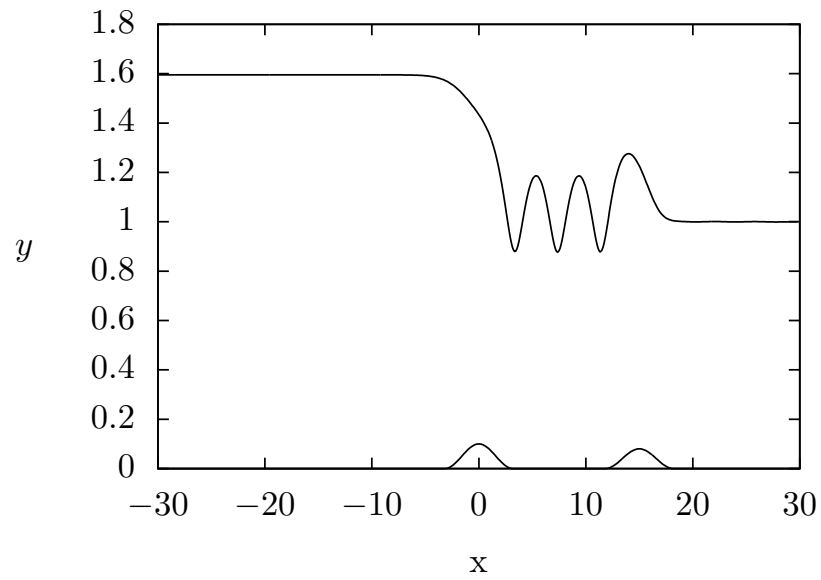

Figure 18: Free-surface profile past 2 submerged obstructions on the bottom of the channel, with surface tension $\tau=0.9$. High amplitude trapped waves are found between the obstacles characterised by $A_{2}=0.05, A_{1}=0.04$ and $L_{1}=L_{2}=3.2$ with $x_{d}=-15$. The Froude number $F=1.4$ is found as part of the solution.

for the parameters $\tau=0.9$ and $F=1.4$. Further, the wavelength of the trapped waves is close to the wavelength predicted by linear theory for gravity capillary waves. The downstream dispersion relation with $\tau=0.9$, shown in Figure 19, predicts waves with a wavenumber of $k \approx 1.68$ for $F=1.4$. The waves seen in Figure 18 are thus expected to have wavelength close to $\lambda \approx 3.74$, and we find that this is indeed the case. Between obstacles centred at $x=0$ and $x=15$, both of half-width 3.2 , we find approximately 2.3 waves, as predicted.

Next we consider hydraulic falls with an additional downstream disturbance of negative orientation; $A_{1}<0$. Again, we find waves trapped between the two disturbances and confirm that the wavelength of the waves is approximately in agreement with the wavelength predicted by linear theory. A near symmetric depression wave is found over the disturbance. The trapped waves in this flow configuration are of smaller amplitude than those in the comparable configuration with $A_{1}>0$. Therefore, for relatively weak tension, or when the height of the first obstacle is large, so that the downstream Froude number $F$ is also large, the free-surface appears to resemble that of a hydraulic fall over the first obstacle, with a near symmetric depression solitary type wave over the downstream disturbance. Figure 20 shows an example free-surface profile for the parameters $A_{2}=0.05, A_{1}=-0.09, L_{1}=L_{2}=3.2, F=1.368$ and $\tau=0.6$. We find that we can compute solutions for much greater $\left|A_{1}\right|$ when $A_{1}<0$, than for the positive case; $A_{1}>0$. As the amplitude of the downstream obstacle gets large however, very small amplitude numerically generated waves appear downstream of the second disturbance. 


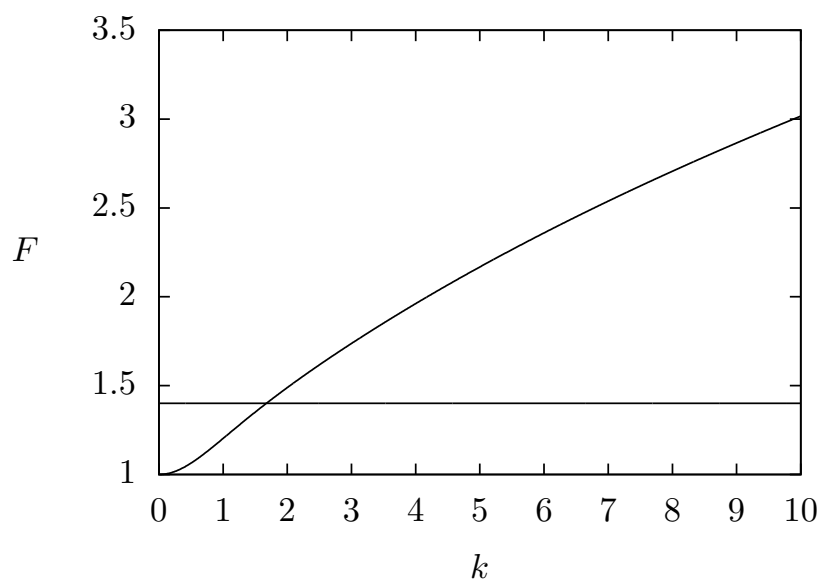

Figure 19: Linear downstream dispersion relation with $\tau=0.9$. The horizontal line $F \approx 1.4$ intersects the dispersion curve at $k \approx 1.68$.

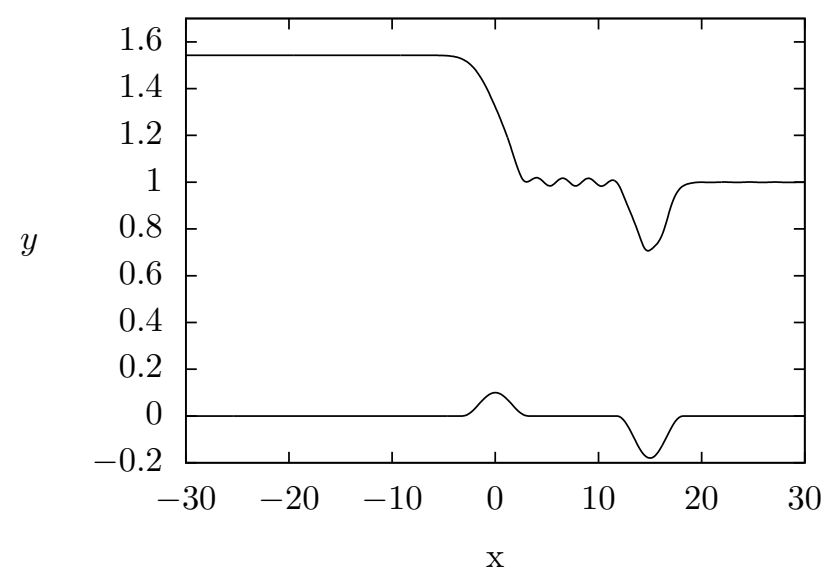

Figure 20: Free-surface profile past 2 submerged obstructions on the bottom of the channel, with surface tension $\tau=0.6$. Trapped waves are found between the obstacles characterised by $A_{2}=0.05, A_{1}=-0.09$ and $L_{1}=L_{2}=3.2$ with $x_{d}=-15$. The Froude number $F=1.368$ is found as part of the solution. 


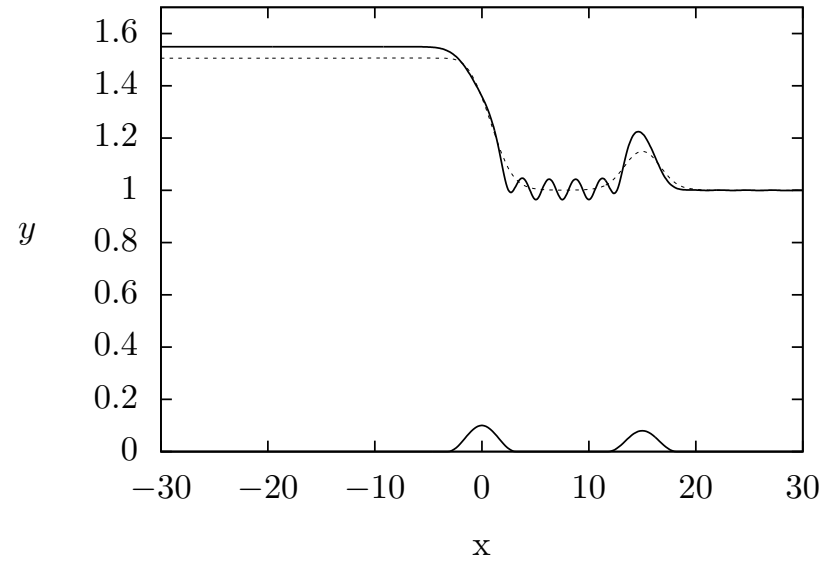

Figure 21: Free-surface profile past 2 submerged obstructions on the bottom of the channel, characterised by $A_{2}=0.05, A_{1}=0.04$ and $L_{1}=L_{2}=3.2$ with $x_{d}=-15$. The solid line is the solution with $\tau=0.6$ and $F=1.372$. Trapped waves are found between the obstacles. The broken curve is the gravity solution with $\tau=0$ and $F=1.345$. The flow appears uniform between the obstacles.

\subsection{Hydraulic falls with a solitary type wave}

Neglecting the effects of surface tension, and setting $x_{d}<0, A_{1}>0$, we obtain solutions with a hydraulic fall over the obstacle centred at $x=0$, and a supercritical solitary type elevation wave over the second obstacle further downstream. Such solutions have previously been obtained by Belward [10], and were discussed briefly in Section 3.3. We showed that in a comparable flow configuration in which the effects of surface tension are included, so long as the tension is strong enough, and the obstacle height $A_{1}$ not too small, a train of trapped waves is found between the obstacles. Here, as with the weaker surface tension cases, the flow between the obstacles appears to be uniform. Figure 21 shows an example free-surface profile alongside the corresponding free-surface profile with strong tension $\tau=0.6$.

In a configuration including the effects of surface tension, solutions with a solitary type wave in addition to the hydraulic fall, can be obtained by setting $x_{d}>0$ so that the second obstacle occurs upstream where the flow is subcritical. So long as the tension is not so small that $F_{\mathrm{up}_{\min }}$, corresponding to the value of the Froude number at the minimum of the upstream dispersion relation, is less than $F_{\text {up }}$, solitary type waves can be found upstream before the hydraulic fall. Solutions with $F_{\text {up }_{\min }}<F_{\text {up }}<1$ have trapped waves between the obstacles, and were discussed in Section 3.3.

A depression solitary wave is found over the upstream obstacle when $A_{1}>0$ and an elevation solitary wave when $A_{1}<0$, with a hydraulic fall over the second obstacle as before. Typical free-surface profiles can be seen in Figure 22. The flow upstream is subcritical, and is comparable with solutions over a 
single obstacle, with subcritical flow, where the effects of surface tension are included. For example, the work of Forbes [11] and Maleewong, Asavanant and Grimshaw $[13,14]$. For strong tension, $\tau>\frac{1}{3}$, Maleewong, Asavanant and Grimshaw [13] obtained a depression solitary wave on a free-surface subjected to a single positively orientated localised pressure distribution, and an elevation solitary wave for a negatively orientated distribution. As $F \rightarrow 1$, the amplitude of the elevation increased. Here, the upstream Froude number is determined predominantly by the height of the obstacle centred at $x=0$. By decreasing the obstacle height, with $\tau_{\text {up }}>\frac{1}{3}$, we increase the upstream Froude number, and see that the amplitude of the elevation increases. Similarly, Maleewong et al. found that as $F$ is increased from zero up to some critical value of $F=F^{*}$, the amplitude of the depression solitary wave also increases. At $F=F^{*}$ their solution branch in the $F-y(0)$ plane, where $y(0)$ is the amplitude of the solitary wave, possesses a turning point, and the solutions change from being perturbations of a uniform stream, to perturbations of a pure solitary wave. We obtain similar results to the perturbations from the uniform stream for the depression wave before the hydraulic fall; by increasing $F_{\text {up }}$, we increase the amplitude of the depression. However, the hydraulic fall requires an extra independent parameter to type 1 solutions, and so we were unable to obtain perturbations from a solitary wave in addition to the hydraulic fall.

For weak tension, the situation is more complicated, because the forced solitary wave solutions of basic type 1 , now resemble envelope solitary waves when the amplitude is small. Maleewong et al. [14] found turning points in the $F-y(0)$ plane for both the elevation and depression solitary waves when $\tau<\frac{1}{3}$. When the forcing is positive, and the Froude number is increased from zero, with $F<F^{*}$, the amplitude of the depression solitary wave increases as with the case $\tau>\frac{1}{3}$. When the forcing is negative and the Froude number is increased from

zero, with $F<F^{* *}, F^{* *}$ a turning point in the $F-y(0)$ plane, the amplitude of the elevation solitary waves increases. These solutions are perturbations from a uniform stream, and we find that decreasing the height of the obstacle centred at $x=0$, which increases the upstream Froude number $F_{\text {up }}$, does increase the amplitude of the elevation wave when $A_{1}<0$, and the depression wave when $A_{1}>0$. Figure 23 shows an example free-surface profile with the solitary type wave before the hydraulic fall. Small oscillations can be seen in the tails of the upstream solitary type waves. As $F$ increases and as $\tau$ decreases, $F$ approaches the minimum of the dispersion curve, and the amplitude and number of the waves in the oscillatory tail, increases. Immediately before the hydraulic fall in Figure 23, a slight elevation in the free-surface can be seen. This compares with the findings of Guayjarernpanishk and Asavanant, discussed in Section 3.1.

\section{Conclusion}

Gravity-capillary forced waves were computed using a numerical method, for the full nonlinear problem. Hydraulic falls were obtained for different values of the surface tension. New types of hydraulic falls are obtained, with decaying oscillations upstream of an obstacle. 


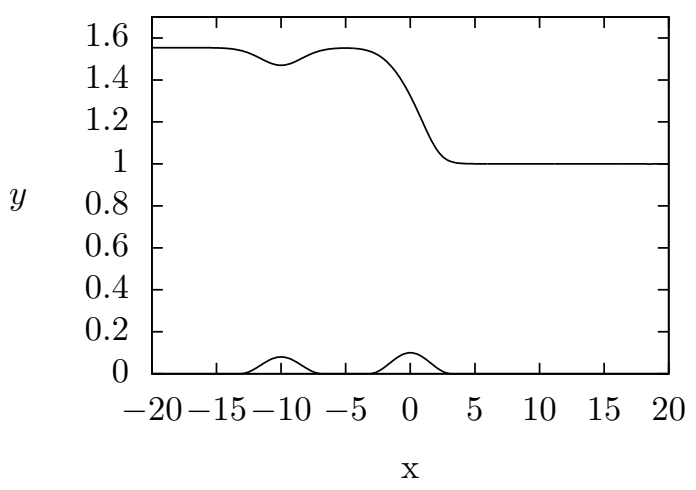

(a)

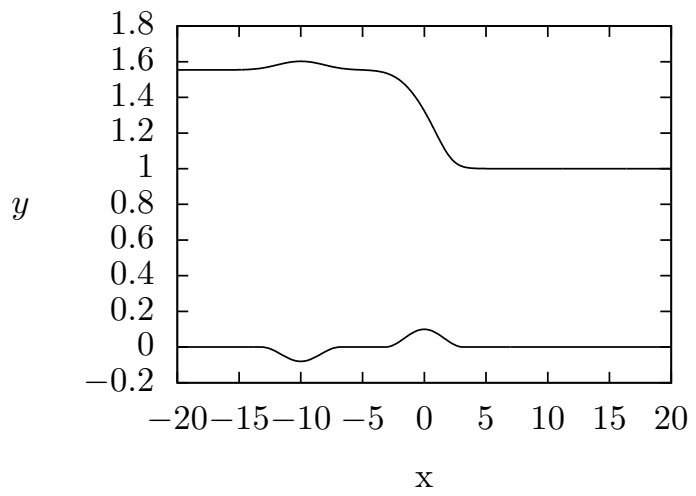

(b)

Figure 22: Free-surface profile past 2 submerged obstructions on the bottom of the channel, with $\tau=0.6$. An elevation wave appears over the upstream obstacle characterised by $A_{1}=$ $\pm 0.04, L_{1}=3.2$ and $x_{d}=10$. The hydraulic fall occurs further downstream over the obstacle characterised by $A_{2}=0.05, L_{2}=3.2$. The value of the Froude number $F=1.375$ is found as part of the solution.

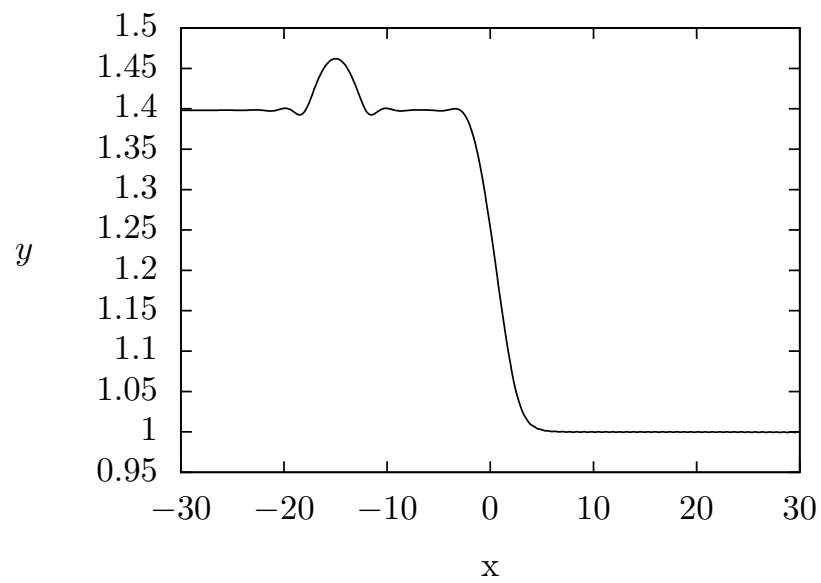

Figure 23: Free-surface profile past 2 submerged obstructions on the bottom of the channel, with $\tau=0.19$. The obstacles are characterised by $A_{1}=-0.03, A_{2}=0.03$ and $L_{1}=L_{2}=3.2$. An elevation solitary wave with damped oscillations appears over the dip before the hydraulic fall. The Froude number $F=1.277$ is found as part of the solution. 
Gravity-capillary generalised hydraulic falls were computed for very small values of the upstream surface tension. However, such solutions lack physical relevance in a flow configuration involving just a single obstacle.

When two submerged obstacles are considered, there are new types of trapped waves between the obstacles, compared with the pure gravity case. These trapped wave solutions are not unique.

The stability of all these flows may be of interest in the future. In the pure gravity case, the stability of some solutions over symmetric obstacles was investigated for model equations in Chardard, Dias, Nguyen and Vanden-Broeck $[20]$.

\section{Acknowledgements}

We would like to thank the anonymous referees for their helpful and constructive comments. 


\section{References}

[1] E. Părău, J.-M. Vanden-Broeck, Nonlinear two- and three-dimensional free surface flows due to moving disturbances, Eur. J. Mech. B/Fluids 21 (2002) $643-656$.

[2] F. Dias, J.-M. Vanden-Broeck, Generalised critical free-surface flows, J. Engrg. Math. 42 (2002) 291-301.

[3] L. Forbes, L. Schwartz, Free-surface flow over a semicircular obstruction, J. Fluid Mech. 114 (1982) 299-314.

[4] J.-M. Vanden-Broeck, Free-surface flow over an obstruction in a channel, Phys. Fluids 30 (1987) 2315-2317.

[5] L. Forbes, Critical free-surface flow over a semi-circular obstruction, J. Engrg. Math. 22 (1988) 3-13.

[6] F. Dias, J.-M. Vanden-Broeck, Open channel flows with submerged obstructions, J. Fluid Mech. 206 (1989) 155-170.

[7] F. Dias, J.-M. Vanden-Broeck, Trapped waves between submerged obstacles, J. Fluid Mech. 509 (2004) 93-102.

[8] L. J. Pratt, On Nonlinear Flow with Multiple Obstructions, J. Atmospheric Sci. 41 (1984) 1214-1225.

[9] B. Binder, F. Dias, J.-M. Vanden-Broeck, Forced solitary waves and fronts past submerged obstacles, Chaos 15 (2005) 037106.

[10] S. Belward, Fully nonlinear flow over successive obstacles: hydraulic fall and supercritical flows, ANZIAM J. 40 (1999) 447-458.

[11] L. Forbes, Free-surface flow over a semicircular obstruction, including the influence of gravity and surface tension, J. Fluid Mech. 127 (1983) 283-297.

[12] S. Grandison, J.-M. Vanden-Broeck, Truncation approximations for gravity-capillary free-surface flows, J. Engrg. Math. 54 (2006) 89-97.

[13] M. Maleewong, J. Asavanant, R. Grimshaw, Free surface flow under gravity and surface tension due to an applied pressure distribtion: I bond number greather than one-third, Theor. Comput. Fluid Dynamics 19 (2005) 237252.

[14] M. Maleewong, R. Grimshaw, J. Asavanant, Free surface flow under gravity and surface tension due to an applied pressure distribtion: Ii bond number less than one-third, Eur. J. Mech. B/Fluids 24 (2005) 502-521.

[15] C. Page, E. Părău, S. Grandison, Gravity-capillary water waves generated by multiple pressure distributions, The Journal of Nonlinear Science and Applications 6 (2013) 137-144. 
[16] P. Guayjarernpanishk, J. Asavanant, Free-surface flows over an obstacle: Problem revisited, in: H. Bock, X. Hoang, R. Rannacher, J. Schlöder (Eds.), Modeling, Simulation and Optimization of Complex Processes, Springer Berlin Heidelberg, 2012, pp. 139-151.

[17] S. Belward, L. Forbes, Fully non-linear two-layer flow over arbitrary topography, J. Engrg. Math. 27 (1993) 419-432.

[18] S. Belward, L. Forbes, Interfacial waves and hydraulic falls: Some applications to atmospheric flows in the lee of mountains, J. Engrg. Math. 29 (1995) 161-179.

[19] F. Dias, J.-M. Vanden-Broeck, Two-layer hydraulic falls over an obstacle, Eur. J. Mech. B/Fluids 23 (2004) $879-898$.

[20] F. Chardard, F. Dias, H. Nguyen, J.-M. Vanden-Broeck, Stability of some stationary solutions to the forced kdv equation with one or two bumps, J. Engrg. Math. 70 (2011) 175-189. 


\section{Figure captions}

Figure1 Dimensionless flow configuration over an arbitrary obstacle on the bottom of the channel.

Figure2 Relationship between the downstream Froude number $F$, and the halfheight $A_{2}$ of the submerged obstacle of half-width $L_{2}=3.2$. The solid curve represents the relationship for $\tau=0$, the dotted curve is the relationship for $\tau=0.2$, and the dashed broken curve is the relationship for $\tau=0.5$. (b) is a close-up of (a), showing the $\tau=0.2$ curve.

Figure3 Hydraulic fall solutions, including the effects of surface tension, over a single submerged obstruction of height $2 A_{2}=0.1$ and half-width $L_{2}=3.2$. The solid line is the solution with $\tau=0.9, F=1.393$, the dashed line the solution with $\tau=0.6, F=1.375$ and the dotted line the solution with $\tau=0.32, F=1.361$. In each case the Froude number was found as part of the solution.

Figure4a Upstream

Figure4b Downstream

Figure4 Relationship between the Bond and Froude number both up and downstream for a single submerged obstacle characterised by $A_{2}=0.05, L_{2}=$ 3.2 .

Figure5 Hydraulic fall solutions, including the effects of surface tension, over a single submerged obstruction of height $2 A_{2}=0.1$ and half-width $L_{2}=$ 3.2 , with Froude number $F=1.398$. The solid line is the solution with $\tau=1.024$, and the broken line is the solution with $\tau=2$. In both cases the Froude number was found as part of the solution.

Figure6 Relationship between the Bond and Froude number downstream for a single submerged obstacles with $L_{2}=3.2$. The dotted line is the branch with $A_{2}=0.01$, the solid line the branch with $A_{2}=0.05$ and the dashed line the branch with $A_{2}=0.1$.

Figure7 Hydraulic fall solution over a single submerged obstruction characterised by $A_{2}=0.015, L_{2}=3.2$. Weak surface tension acts on the freesurface; $\tau=0.2$. A small train of decaying waves immediately before the hydraulic fall can be seen. The Froude number $F=1.198$ is found as part of the solution.

Figure8 Generalised hydraulic fall solution over a submerged obstruction of height $2 A_{2}=0.2$ and half-width $L_{2}=3$. The downstream Froude and Bond numbers are given by $F=1.482$ and $\tau=0.01$ respectively.

Figure9a Pure gravity case, $\tau=0$.

Figure9b Gravity-capillary case, $\tau=0.5$. 
Figure9 Linear dispersion relations. The solid curves represent the dispersion relation downstream, and the solid constant lines give the downstream value of the Froude number $F$ for a submerged obstacle classified by $A_{2}=0.05, L_{2}=3.2$. The broken curves represent the dispersion relation upstream, and the broken constant lines give the upstream value of the Froude number $F_{\text {up }}$ for the same obstacle.

Figure10 Free-surface profile past 2 disturbances on the bottom of the channel. The first disturbance represents a dip in the channel, and is given by a) $A_{1}=-0.05, L_{1}=3.2$, b) $A_{1}=-0.3, L_{1}=3.2$, centred upstream at $x=$ -15 , and the second is an obstacle characterised by $A_{2}=0.05, L_{2}=3.2$. Waves appear trapped between the obstacles. In a) $F=1.348$ and an elevation wave is found over the dip. In b) $F=1.364$ and a two-peaked elevation wave is found over the dip. In both cases, the Froude number was found as part of the solution.

Figure11 Free-surface profile past 2 obstacles on the bottom of the channel, with weak tension $\tau=0.1$. The first of which is characterised by $A_{1}=$ $0.02, L_{1}=3.2$ and is centred upstream at $x=-15$, and the second by $A_{2}=0.1, L_{2}=3.2$. Small amplitude waves appear trapped between the obstacles. The Froude number $F=1.497$ is found as part of the solution.

Figure12 Free-surface profile past 2 obstacles on the bottom of the channel, characterised by $A_{2}=0.05, L_{2}=3.2$ and $A_{1}=0.01, L_{1}=3.2$. The solid curve represents the solution with $\tau=0.1$ and $F=1.350$. The broken curve represents the pure gravity solution with $\tau=0$ and $F=1.334$.

Figure13 Upstream linear dispersion relations. The solid curves represent the dispersion relation with $\tau=0.1$ and the upstream value of the Froude number $F_{\text {up }}$ for submerged obstacles characterised by $A_{2}=0.05, L_{2}=3.2$ and $A_{1}=0.01, L_{1}=3.2$. The broken curves are the dispersion relation with $\tau=0$ and the upstream value of the Froude number for the same channel bottom configuration.

Figure14 Free-surface profile past 2 obstacles on the bottom of the channel, with surface tension $\tau=0.6$. The first of which is characterised by $A_{2}=$ $0.05, L_{2}=3.2$ and the second by $A_{1}=0.04, L_{1}=3.2$, centred downstream at $x=15$. Trapped waves appear trapped between the obstacles. The Froude number $F=1.372$ is found as part of the solution.

Figure15 Free-surface profile past 2 obstacles on the bottom of the channel, with surface tension $\tau=0.4$. The first of which is characterised by $A_{2}=$ $0.1, L_{2}=3.2$, and the second $A_{1}=0.02, L_{1}=3.2$, centred downstream at $x=15$. The free-surface appears to be uniform between the obstacles. The Froude number $F=1.512$ is found as part of the solution.

Figure16a Upstream

Figure16b Downstream 
Figure16 Gravity-capillary trapped wave solution branches in a) the $F_{\text {up }}-\tau_{\text {up }}$ plane and b) the $F-\tau$ plane. The two submerged obstacles on the bottom of the channel are classified by $A_{2}=0.1, A_{1}=0.05$ and $L_{1}=L_{2}=3.2$.

Figure17a Solutions for $\tau=0.8, \tau=1.2074$ and $\tau=1.54212$

Figure17b Solutions for $\tau=1.74, \tau=2.973$ and $\tau=4.451$

Figure17 Free-surface profiles past two obstacles on the bottom of the channel, characterised by $A_{2}=0.1, L_{2}=3.2$, centred at $x=0$ and $A_{1}=0.05$, $L_{1}=3.2$ centred downstream at $x=15$. Multiple trapped wave solutions are found for the same value of the Froude number $F=1.53597$, but with different values of the Bond number. For clarity, only the central part of the free-surface is shown.

Figure18 Free-surface profile past 2 submerged obstructions on the bottom of the channel, with surface tension $\tau=0.9$. High amplitude trapped waves are found between the obstacles characterised by $A_{2}=0.05, A_{1}=0.04$ and $L_{1}=L_{2}=3.2$ with $x_{d}=-15$. The Froude number $F=1.4$ is found as part of the solution.

Figure19 Linear downstream dispersion relation with $\tau=0.9$. The horizontal line $F \approx 1.4$ intersects the dispersion curve at $k \approx 1.68$.

Figure20 Free-surface profile past 2 submerged obstructions on the bottom of the channel, with surface tension $\tau=0.6$. Trapped waves are found between the obstacles characterised by $A_{2}=0.05, A_{1}=-0.09$ and $L_{1}=$ $L_{2}=3.2$ with $x_{d}=-15$. The Froude number $F=1.368$ is found as part of the solution.

Figure21 Free-surface profile past 2 submerged obstructions on the bottom of the channel, characterised by $A_{2}=0.05, A_{1}=0.04$ and $L_{1}=L_{2}=3.2$ with $x_{d}=-15$. The solid line is the solution with $\tau=0.6$ and $F=1.372$. Trapped waves are found between the obstacles. The broken curve is the gravity solution with $\tau=0$ and $F=1.345$. The flow appears uniform between the obstacles.

Figure22 Free-surface profile past 2 submerged obstructions on the bottom of the channel, with $\tau=0.6$. An elevation wave appears over the upstream obstacle characterised by $A_{1}= \pm 0.04, L_{1}=3.2$ and $x_{d}=10$. The hydraulic fall occurs further downstream over the obstacle characterised by $A_{2}=0.05, L_{2}=3.2$. The value of the Froude number $F=1.375$ is found as part of the solution.

Figure23 Free-surface profile past 2 submerged obstructions on the bottom of the channel, with $\tau=0.19$. The obstacles are characterised by $A_{1}=$ $-0.03, A_{2}=0.03$ and $L_{1}=L_{2}=3.2$. An elevation solitary wave with damped oscillations appears over the dip before the hydraulic fall. The Froude number $F=1.277$ is found as part of the solution. 